\title{
Use of diffusion tensor imaging in glioma resection
}

\author{
Kalil G. Abdullah, M.D., ${ }^{1}$ Daniel Lubelski, B.A., ${ }^{2}$ Paolo G. P. Nucifora, M.D., Ph.D., ${ }^{3}$ \\ and STEven BRem, M.D. ${ }^{1}$
}

\author{
${ }^{1}$ Department of Neurosurgery; ${ }^{3}$ Department of Radiology, Section of Neuroradiology, Hospital of the \\ University of Pennsylvania, Philadelphia, Pennsylvania; and ${ }^{2}$ Cleveland Clinic Lerner College of Medicine, \\ Cleveland, Ohio
}

\begin{abstract}
Diffusion tensor imaging (DTI) is increasingly used in the resection of both high- and low-grade gliomas. Whereas conventional MRI techniques provide only anatomical information, DTI offers data on CNS connectivity by enabling visualization of important white matter tracts in the brain. Importantly, DTI allows neurosurgeons to better guide their surgical approach and resection. Here, the authors review basic scientific principles of DTI, include a primer on the technology and image acquisition, and outline the modality's evolution as a frequently used tool for glioma resection. Current literature supporting its use is summarized, highlighting important clinical studies on the application of DTI in preoperative planning for glioma resection, preoperative diagnosis, and postoperative outcomes. The authors conclude with a review of future directions for this technology. (http://thejns.org/doi/abs/10.3171/2013.1.FOCUS12412)
\end{abstract}

KEY WoRds $\quad$ brain mapping • diffusion tensor imaging • glioma
connectome

$\mathrm{D}$ IFFUSION tensor imaging is a form of diffusionweighted MRI that assesses physiological water directionality and motion, providing images of important white matter tracts within the CNS. ${ }^{25}$ Conventional MRI techniques provide purely anatomical information without data regarding CNS connectivity. The ability to visualize important white matter tracts in the brain enables neurosurgeons to better guide their surgical approach and resection. Below, we provide a primer on the biophysical basis of DTI, review the current state of the literature on the use of DTI for glioma resection, and discuss future advances in the field.

\section{Technical and Biophysical Considerations of DTI}

Various cellular structures-for example, cell membranes and intracellular organelles-impede the random motion of water molecules in the brain and instead cause them to move with some form of directionality called "anisotropy" (Table 1). ${ }^{41,42}$ This biological property is

Abbreviations used in this paper: $\mathrm{ADC}=$ apparent diffusion coefficient; DTI = diffusion tensor imaging; EPI = echo planar imaging; $\mathrm{FA}=$ fractional anisotropy; fMRI = functional MRI; GBM = glioblastoma multiforme; GTR = gross-total resection; KPS = Karnofsky Performance Scale; MD = mean diffusivity; ROI = region of interest; SLF = superior longitudinal (arcuate) fasciculus. essential to understanding DTI, because the directionality of water molecules as they move within white matter tracts is a key component of fiber tracking. The orientation of white matter tracts causes anisotropy, because water diffuses in a direction parallel to the axonal fibers as a result of the myelin sheaths, which create a barrier to the diffusion of water perpendicular to the axonal membranes. ${ }^{1,9,30,31}$ Collectively, this information is known as the "diffusion tensor," a 3D ellipsoid model of water diffusion. The diffusion tensor directly represents the direction (anisotropy) of water and indirectly represents the orientation of white matter fibers. ${ }^{25}$ It is described as a 3D ellipsoid and is subjected to a linear algebraic procedure known as "diagonalization." The diffusion tensor can then be represented by 3 eigenvalues $\left(\lambda_{1} \geq \lambda_{2} \geq \lambda_{3}\right)$, which are measures of the magnitude of diffusion, and subsequently by 3 eigenvectors $\left(\mathrm{v}_{1}, \mathrm{v}_{2}, \mathrm{v}_{3}\right)$, which are orthogonal to each other and represent the direction of diffusion. ${ }^{9,25}$ The metrics for DTI are FA and MD. Fractional anisotropy is the measurement of the tendency of water to diffuse in one direction (anisotropy), whereas MD measures the magnitude of diffusion. Mean diffusivity is comparable and mathematically equivalent to the ADC in standard diffusion-weighted imaging. It is calculated as the mean of the 3 eigenvalues, representing the directionally averaged diffusivity of water, which is affected by changes in the structure of brain tissue. ${ }^{21}$ Fractional 


\begin{tabular}{|c|c|}
\hline Term & Definition \\
\hline anisotropy & directional dependence of water; properties have different values depending on their direction/orientation \\
\hline isotropy & $\begin{array}{l}\text { properties are the same in all directions; random motion of water molecules in all directions } w / \text { no preferential direc- } \\
\text { tion }\end{array}$ \\
\hline diffusion tensor & $\begin{array}{l}\text { 3D mathematical ellipsoid model of water diffusion; directly represents directionality of water, indirectly represents } \\
\text { white matter fibers }\end{array}$ \\
\hline diagonalization & linear algebraic manipulation of diffusion tensor dimensions used to calculate eigenvectors \\
\hline eigenvector & measure of the direction of diffusion \\
\hline eigenvalue & measure of the magnitude of diffusion \\
\hline fractional anisotropy & $\begin{array}{l}\text { measure/degree of anisotropic water diffusion, calculated from the eigenvalues \& ranging from } 0 \text { (maximal isotropy) } \\
\text { to } 1 \text { (maximal anisotropy) }\end{array}$ \\
\hline mean diffusivity & directionally averaged diffusivity of water; mean of all eigenvalues \\
\hline apparent diffusion coefficient & $\begin{array}{l}\text { diffusion constant, expressed as } \mathrm{mm}^{2} / \mathrm{sec} \text {, relates average displacement of a water molecule over a specific area } \\
\text { over time; greater values indicate greater water mobility }\end{array}$ \\
\hline DTI b-value & $\begin{array}{l}\text { degree of diffusion weighting, determined by the sensitizing gradient scheme (strength, duration, \& temporal spac- } \\
\text { ing), typically set between } 700 \text { and } 1200 \mathrm{sec} / \mathrm{mm}^{2}\end{array}$ \\
\hline
\end{tabular}

anisotropy is calculated from the standard deviation of the 3 eigenvalues, ranging from 0 (isotropy with 0 net direction) to 1 (maximum anisotropy along 1 eigenvector). ${ }^{11}$ This directionality is typically presented in a color-coded map or via fiber tractography whereby the color hue indicates directionality and brightness is proportional to the FA. ${ }^{35}$ A general convention is to color code the projection fibers (blue) coursing from superior to inferior (for example, the corticospinal tract), the association fibers (green) coursing anterior to posterior (for example, the arcuate fasciculus), and the commissural fibers (red) coursing laterally (for example, the corpus callosum).

Images are acquired using a clinical MRI unit (1.5- or 3-T systems) with a standard head coil. Diffusion tensor imaging is performed using a single-shot EPI sequence. Echo planar imaging is a fast acquisition technique that reduces motion-related artifacts. Single-shot EPI is commonly used instead of multishot EPI despite its poorer spatial resolution, because it has a shorter acquisition time, a superior signal-to-noise ratio, and less motionrelated distortion. ${ }^{1}$ These techniques have enabled a fast acquisition time of less than 5 minutes. ${ }^{52}$ The degree of diffusion weighting, represented as the b-value, is determined by a sensitizing gradient scheme composed of strength, duration, and temporal spacing. ${ }^{38}$ Diffusion gradient weighting can be done in as few as 6 orientations/ directions and in as many as 512, which is the capacity of many imaging systems. Most groups acquire between 6 and 55 directions, ${ }^{21}$ with more encoding directions increasing the required scan time but decreasing the variance in tensor model parameters ${ }^{25}$ and the signal-to-noise ratio. 38

Diffusion tensor imaging measurements are obtained using either ROI analysis or tractography. Region of interest analysis is based on operator experience and is sometimes difficult to reproduce in areas with brain tumors or edema. ${ }^{44}$ Frequently, DTI tractography can be performed first to localize the tracts, and then ROI analysis can be used based on the previously identified tracts, thereby re- ducing selection biases of the ROI. ${ }^{35}$ Tractography can be performed via the deterministic or probabilistic method. The deterministic method initiates fiber trajectories using fiber assignment by continuous tracking (FACT) of userdefined voxels. ${ }^{7,31}$ Various factors, including noise, patient movement, and image artifact, can create uncertainty in DTI measurements; ${ }^{31}$ these uncertainties can be adjusted for by using the probabilistic method, in which an additional quantification of the probability of connection between 2 points is performed, allowing the depiction of a greater portion of white matter tracts. The probabilistic method is advantageous in areas of lower anisotropy, such as small tracts and crossing fibers, as well as in gray matter. ${ }^{2}$

White matter fiber tracts are traditionally divided into association fibers that connect cortical areas within the hemisphere; projection fibers that connect cortical areas to the deep nuclei, brainstem, cerebellum, and spinal cord; and commissural fibers that interconnect similar cortical areas of opposite hemispheres. The association fibers commonly visualized on DTI are the cingulum, superior and inferior frontooccipital fasciculus, uncinate fasciculus, SLF, and inferior longitudinal (occipitotemporal) fasciculus. The projection fibers visualized on DTI include the corticospinal, corticobulbar, and corticopontine projection fibers; the commissural fibers seen on DTI include the corpus callosum and anterior commissure. In certain cases, other less commonly used tracts may be identified, such as the optic pathways or fiber tracts within the brainstem. . $^{18,23,29,45}$

\section{Evolution of DTI for Neurosurgical Use}

The first authors to integrate the use of fiber tracking into intraoperative navigation were Coenen and colleagues in $2001^{10}$ in 4 patients undergoing resection of both intra- and extraaxial tumors (2 glioblastomas and 2 Grade I meningiomas). Diffusion-weighted, not diffusion tensor, imaging was used to visualize the pyramidal tracts for intraoperative navigation. Several studies fol- 
lowed, and most investigators applied direct stimulation to verify the fidelity of DTI fiber tracking. In 2003, Kamada et al. ${ }^{27}$ used DTI and direct fiber stimulation to confirm the accuracy of DTI in the intraoperative identification of the corticospinal tract in 6 patients with intraaxial lesions. In 2004, Berman et al. ${ }^{4}$ combined DTI and direct cortical stimulation in 11 patients undergoing glioma resection. These investigators found that DTI fiber tracts maintained a high degree of correlation with the descent of motor tracts from the cortex to the cerebral peduncle. This same group also reported on in vivo detection of white matter tracts involved in speech and naming by using DTI derived from direct cortical stimulation during resection of a left frontotemporal glioma. ${ }^{22}$

In 2005, Nimsky et al. ${ }^{34}$ described 37 patients undergoing supratentorial glioma surgery in whom both preand intraoperative DTI was performed. These authors analyzed white matter tract shifting and bidirectional (outward and inward) shifting of tracts. They found that the amount of white matter tract shift corresponded well with brain shift of the "deep tumor margin," as previously described by Dorward et al. ${ }^{12}$ At the time, the study by Nimsky et al. ${ }^{34}$ represented one of the largest series of patients to undergo glioma resection aided by DTI and one of the first series to do so without the concurrent use of direct cortical stimulation. This same group of authors later performed additional studies that advanced the earlier techniques. They first described 19 more patients undergoing glioma resection with the integration of both fMRI and DTI ${ }^{35}$ and then 16 patients to demonstrate fiber tracking in standard neuronavigational systems. ${ }^{33}$ They also compared preoperative fiber tracts with intraoperative shifting of the fiber tracts. ${ }^{34}$

In 2007 , Wu et al..$^{50}$ performed a rare prospective randomized controlled trial to evaluate the impact of DTI versus standard neuronavigation in patients with gliomas involving the pyramidal tracts. These authors reported on a consecutive series of 238 patients, with 118 of them randomized to a group undergoing DTI fiber tracking integrated into neuronavigation during resection and 120 randomized to a group undergoing standard MRI protocols and standard neuronavigation. Follow-ups lasted up to 50.5 months (median 21.3 months). The 6-month KPS scores were assessed in $96.34 \%$ of enrolled patients. The KPS scores among patients with either low- or high-grade gliomas were significantly higher in those who had undergone DTI than in those who had undergone standard neuronavigational resection: overall, for patients with gliomas, 86 vs 74, p < 0.001; for those with low-grade gliomas, 93 vs 86, $\mathrm{p}=0.013$; and for those with highgrade gliomas, 77 vs 53, $\mathrm{p}=0.001$. Survival analysis in the subset of 81 patients with high-grade gliomas (including GBM, anaplastic astrocytomas, anaplastic oligodendrogliomas, and anaplastic ependymomas) showed a significant survival benefit in those who had undergone DTIassisted tumor resection (median survival 21.2 months) as compared with the control group (median survival 14.0 months, $p=0.048$ ). When analyzing survival curves in the study, the survival benefit was particularly pronounced in the early to mid-postoperative period (until 30 months), a time period when the surgical technique would be ex- pected to have the greatest impact on survival outcome. In particular, for a subset of 50 patients with glioblastoma or gliosarcoma (WHO Grade IV), there was a significant difference in median survival of 19.3 months (DTI group) versus 11.2 months (control group, $\mathrm{p}=0.02$ ), representing a $53.6 \%$ reduction in the risk of death over the course of the study. ${ }^{53}$ A separate analysis of only WHO Grade IV neoplasms showed a similarly statistically significant survival benefit among patients undergoing DTI-aided resection (19.3 vs 11.2 months). Additionally, the overall rates of GTR in the DTI group (118 cases, 72\% GTR) were significantly higher than in the control group that underwent standard neuronavigation (120 cases, 51.7\% GTR, $\mathrm{p}=0.002$ ).

This study is a key component of the neurosurgical literature for several reasons. First, it is one of very few Class I (prospective randomized controlled) studies to link outcomes of glioma surgery with neurosurgical technique. Second, it shows clear benefits in increasing overall survival, extent of resection, and 6-month KPS score in the DTI-aided high-grade glioma group. However, the study does have a few limitations. 1) Progressionfree survival rates for patients undergoing resection of low-grade gliomas were not analyzed, and a neurological outcome benefit among patients with low-grade gliomas was not demonstrated. 2) The baseline KPS scores for patients undergoing high-grade glioma resection were not provided, and a high preoperative KPS score is known to be a strong prognostic factor for survival. Despite these drawbacks, the study remains a key piece of evidence supporting the use of DTI-aided resection of gliomas.

Combining DTI with subcortical mapping and direct subcortical stimulation has also been investigated. Bello et al. ${ }^{3}$ reported the results for 230 patients with glioma who had undergone DTI and subcortical mapping of the corticospinal and language tracts (SLF, inferior frontooccipital fasciculus, uncinate fasciculus, and inferior longitudinal [occipitotemporal] fasciculus). Using a combination of direct stimulation and DTI, these authors found the preservation of language function in $96.8 \%$ of patients who had lesions in areas or pathways involved in speech function, as well as preserved motor function in 94\% of patients. The authors emphasized the dual roles of DTI and cortical stimulation; the former offers information about anatomical relationships, whereas the latter provides information about functionality. This distinction between structure and function is particularly important in the resection of tumors in language areas, as anatomical tract distributions (for example, the SLF) can often be broader than their functionally relevant counterparts. ${ }^{3}$

Integrating anatomical and functional information is particularly important. Multimodal navigation, as it refers to lesion localization and resection assistance, may include direct cortical and subcortical stimulation along with fMRI and DTI sequences. Often these imaging modalities can be used simultaneously and allow for intraoperative overlay of both functional and anatomical spatial relationships (Figs. 1 and 2). This combined approach was used by González-Darder and colleagues ${ }^{19}$ in 17 patients who underwent resection of gliomas in motor areas with the aid of DTI, cortical and subcortical map- 

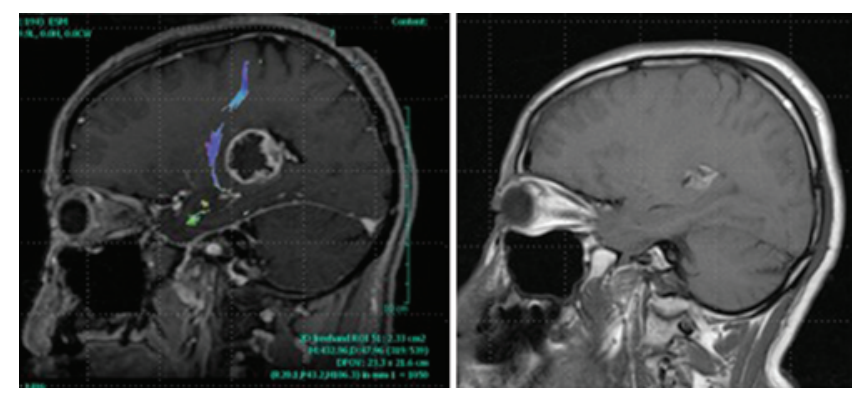

FIG. 1. Preoperative DTI with corticospinal tract mapping in the case of a thalamic GBM. Preoperative sagittal T1-weighted postcontrast STEALTH MR image with DTI of the corticospinal tract (left) and sagittal T1-weighted MR image obtained on postoperative Day 8 (right). Images were obtained in a 54-year-old right-handed man who presented with an episode of severe headache. The mass measured $3.7 \times 2.6 \mathrm{~cm}$ and was centered in the right thalamus, causing a $6-\mathrm{mm}$ midline shift. For mapping, the seed ROls were placed at the cerebral peduncles. The patient was discharged on postoperative Day 3 without neurological deficit.

ping, and fMRI. As in other studies, a high degree of correlation existed between cortical points tested with direct stimulation and the density of motor fibers as evidenced by DTI. Using the multimodality approach, they noted that on routine anatomical (traditional intraoperative visual) inspection, the central sulcus and precentral gyrus were correctly identified in only 11 of 17 cases. Functional MRI without direct stimulation was not helpful in identifying these areas, because of the signal distortion of underlying tumor. With the addition of DTI, however, the central sulcus and precentral gyrus were easily apparent in all cases. While end points such as maximal resection and progression-free survival are important, the more basic task of identifying key anatomical landmarks has been shown to be aided greatly by tractography.

Recent studies have continued to validate concordance between DTI and subcortical mapping. ${ }^{36,37,55}$ In 2012, Ohue et al. ${ }^{37}$ examined the accuracy of DTI in 32 patients with
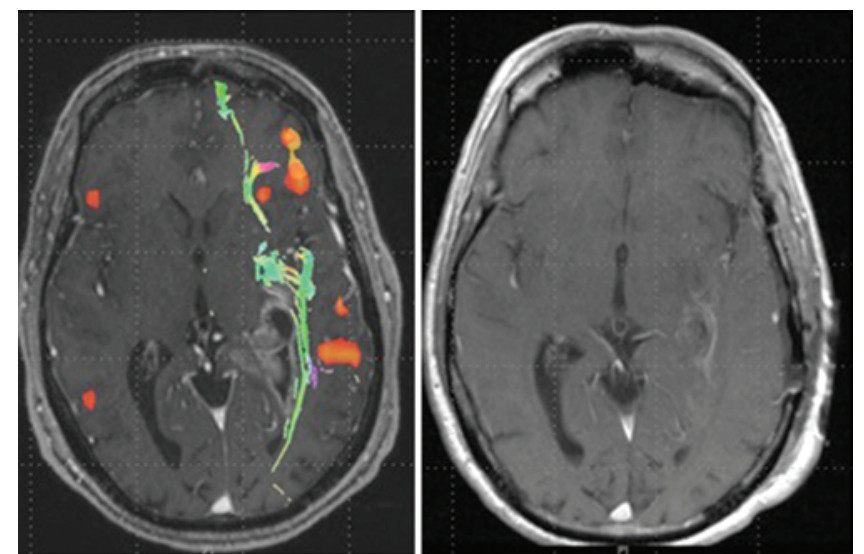

FIG. 2. Preoperative DTI of the SLF and superimposed $f M R I$ in a temporal lobe GBM. Preoperative axial postcontrast STEALH DTI of the SLF and superimposed fMRI (left) and postoperative postcontrast image (right). Images were obtained in a 70-year-old right-handed man with short-term memory loss, word-finding difficulties, and fatigue of 3 months' duration. He underwent a left temporal awake craniotomy and was discharged home on postoperative Day 3 with no new neurological deficits. At the 1-month follow-up there were resolution of fatigue and improvements in word finding. gliomas near the pyramidal tract by using cortical and subcortical motor evoked potentials. Additionally, Zhu et al..$^{55}$ conducted a prospective study of 58 patients with gliomas in proximity to the pyramidal tract. These authors found high concordance between DTI representation of the pyramidal tracts and subcortical mapping, with sensitivity and specificity over $90 \%$ for DTI. The distance between preoperatively imaged diffusion tensor tracts and those found intraoperatively via direct stimulation averaged $5.2 \mathrm{~mm}$, with a range from 2.0 to $14.7 \mathrm{~mm}$. While still supporting the validity of DTI, this study as well as others reinforces the importance of accounting for brain shift when using DTI for navigation. Recently, Ius et al. ${ }^{24}$ documented results in 190 patients who had undergone resection of lowgrade gliomas in eloquent areas. While all of the patients underwent direct subcortical stimulation, a subset of 117 more contemporary patients underwent resection along with DTI and fMRI. The extent of resection was significantly greater in patients who had undergone DTI- and fMRI-incorporated neuronavigation (90\% vs $77 \%$ ). Additionally, while subcortical stimulation assists in the avoidance of direct trauma to relevant fiber tracts, it does not provide visual warning of vascular damage that may occur to the periphery of those tracts, a clear benefit of DTI and, to some extent, fMRI.

\section{Use of DTI in Uncommon Gliomas}

Although cortical and subcortical gliomas have been the focus of most studies examining the use of DTI in tumor resection, several investigators have demonstrated the promise of DTI in resecting less frequently occurring gliomas. Recent reports have shown the value of DTI for resections near the optic pathway and radiations. ${ }^{29,32,45}$ Lober et al. ${ }^{29}$ described 10 patients with optic gliomas, in whom they were able to successfully visualize tractography and provide correlation with preoperative clinical symptoms, including an array of specific arrangements of visual fibers dependent on the prechiasmatic or chiasmatic location of the tumors. Sun et al. ${ }^{45}$ used DTI for visualization of the optic radiations in 43 patients and found that the surgical approach should be modified in 6 patients.

Interestingly, Setzer et al. ${ }^{43}$ examined the value of DTI in patients with intramedullary spinal cord tumors. They evaluated 14 patients and preoperatively classified their tumors as resectable or unresectable based on the fiber course in relation to the lesion. They classified lesions as Types I-III: Type I, fibers did not enter the lesion; Type II, some fibers entered the lesion; and Type III, most of the fibers entered the lesion or were destroyed by the lesion. These findings were then compared with the intraoperative plane of dissection-a reliable marker of the resectability of intramedullary spinal cord tumor. All Type I tumors were successfully resected. Among the 6 Type III tumors, 5 were considered unresectable and 1 with a clear resection plane intraoperatively was grosstotally resected. Unfortunately, the patient in this last case ultimately experienced a clinical deterioration postoperatively. While these results are certainly interesting, they are tempered by a small sample size, making it difficult to extrapolate from the results. 


\section{From Topology to Hodotopy}

There has been substantial interest in and advocacy for increased attention on the concept of brain "hodotopy," a term derived from the classic neuroscientific principal of hodology (from the Greek hodos, the study of pathways). ${ }^{17}$ This concept, first brought to attention by Catani $^{6}$ and Duffau, ${ }^{13,14}$ has been described as a "dynamic organization of the CNS constituted by parallel distributed networks." This novel paradigm implies that these networks have substantial subcortical connections that, when left preserved, allow reorganization over time. The practical aspect of this approach is that multistage resections in eloquent areas allow improved resection once functional remapping has occurred. ${ }^{40}$ Neuroplasticity can be of particular importance in the resection of low-grade gliomas, in which tumor cells have been found far outside the boundaries of perceived maximal resection. When low-grade gliomas occur in eloquent areas, the ability to achieve a supramaximal resection (tumor + margin) to limit tumor recurrence from isolated, dispersed glioma cells is substantially limited.

"Supratotal" resection of WHO Grade II gliomas using intraoperative awake direct stimulation was shown to be effective, as compared with only complete resection in a control group. ${ }^{54}$ Importantly, even though adjuvant treatment was given to 10 patients in the control group (vs only 1 in the supratotal resection group), anaplastic transformation occurred in 7 of them (vs none in the experimental group). While the findings were encouraging and statistically significant $(\mathrm{p}=0.043)$, the study included relatively few (15) patients. Note that the goal of supracomplete resection is to delay anaplastic transformation of low-grade gliomas. A promising frontier in the neurosciences is that reorganization of the white matter pathways over time can be better understood after neurosurgical resections with tractography. ${ }^{53}$ Additionally, these concerns are currently most cogently applied to the resection of low-grade gliomas (as opposed to high-grade gliomas), whose natural history allows for longitudinal studies of brain reorganization and plasticity. Regardless, the cited studies provide a strong basis for further investigation into the neurosurgical examination of long-term neural network connectivity.

\section{Diffusion Tensor Imaging for Preoperative Diagnosis}

Many authors have examined the use of DTI to distinguish gliomas from other pathologies, to predict the extent of resection, and to understand posttreatment changes to white matter. $5,8,18,23,26,28,39,41,44,46,48,49,51,56$ The ability to differentiate brain gliomas, metastases, and lymphomas has been a source of interest since the advent of advanced neuroimaging. For DTI, factors such as FA, linear anisotropy coefficients, cerebral blood volume, ADCs, and other diffusion tensor indices have all been implicated in differentiating high-grade gliomas and other lesions that have similar visual characteristics on standard MRI sequences. Diffusion tensor imaging in conjunction with MR spectroscopy, ADCs, and other MRI protocols provides highly specific and sensitive information for diagnosis. Many of the above-cited studies have extremely low Type I error rates, with sensitivities and specificities greater than $90 \%$. Advanced neuroimaging may aid in the preoperative estimation of pathology, but the gold standard remains histological analysis.

\section{Future Directions of DTI}

The ability to visualize relevant white matter tracts during glioma resection represents an important advance in the treatment of brain tumors. While neuronavigation has substantially improved the ability of neurosurgeons to maneuver within eloquent areas of the brain, DTI has already proved a powerful tool in the neurosurgeon's armamentarium. This imaging modality's transition from the basic science laboratory to clinical use has followed an accelerated course. In 1999, Conturo et al. ${ }^{11}$ presented the first known instance of tracking neuronal fiber pathways in a living human. Three years later, Gössl et al. ${ }^{20}$ briefly discussed its possible neurosurgical use, and only 1 year later Duffau et al. ${ }^{16}$ referenced DTI as a possible avenue of research in their analysis of 103 patients with gliomas treated with resection guided by direct stimulation. Within the next 2 years, several groups began investigating the integrity of DTI and subsequently its use in tumor resection. ${ }^{22,27,29,35}$ Diffusion tensor imaging has become a more widely used tool at many centers. At our center, DTI (often in conjunction with fMRI, subcortical stimulation, and cortical and awake mapping) is routinely performed for all tumors within or near the motor and language areas (Figs. 1 and 2).

Several components of DTI represent future challenges. The first and most obvious challenge is brain shift, which remains an issue under investigation. Preoperative DTI mapping has been shown to have relatively high fidelity, but inward and outward shift occurs after the dura mater is opened, and some centers still concurrently use direct stimulation, ultrasound, or intraoperative MRI to account for these changes. Additionally, tracts are often disrupted by the pathology of interest. Gliomas or other lesions can disrupt or distort fiber tracts by invasion or vasogenic edema, resulting in a lowering of the anisotropy (FA) value. The confounding effect of complex glioma biology is an intrinsic problem with DTI and supports the use of multimodal approaches for glioma resection.

Several technologies seem poised to integrate with DTI and advance the scope of neuronavigation. First, the resolution of imaging that is the basis of DTI has become increasingly robust. High angular resolution diffusion imaging (HARDI) represents one of these technologies. It allows the resolution of crossing fibers, providing a more precise and thorough visualization of fibers in any given region of interest. Another avenue of interest is the developing field of connectomics, which aims to provide a blueprint for the totality of neural networks by elucidating individual neuronal connections. The ramifications of a better understanding of neural networks is clear. Diffusion tensor imaging has provided excellent resolution of main fiber pathways involved in functional activities: speech, vision, and motor. Connectomics is 
an attempt to understand these and other processes at a more basic level. ${ }^{47}$ The initiative known as the Human Connectome Project, supported by the National Institutes of Health, could transform neurosurgical oncology. This nascent field heavily utilizes advanced imaging, such as HARDI, and has already begun to reveal subtle features of brain connectivity not well visualized with DTI. The application of connectomics is even more topical when taking into account the recent enthusiasm for a hodotopic approach to the resection of brain lesions, particularly gliomas. ${ }^{13-15,40}$ The ability to better appreciate long-term connectivity of the CNS in the setting of resection will clearly provide more insight into the best management and practice.

Diffusion tensor imaging has become a cornerstone in the resection of gliomas in or near eloquent areas. With future research and development, this imaging modality may be further understood and optimized, and advanced imaging techniques and a better understanding of neural connections will continue to advance the neurosurgeon's ability to optimize patient outcomes.

\section{Disclosure}

The authors report no conflict of interest concerning the materials or methods used in this study or the findings specified in this paper.

Author contributions to the study and manuscript preparation include the following. Conception and design: all authors. Acquisition of data: Brem, Abdullah, Lubelski. Analysis and interpretation of data: all authors. Drafting the article: all authors. Critically revising the article: all authors. Reviewed submitted version of manuscript: all authors. Approved the final version of the manuscript on behalf of all authors: Brem.

\section{References}

1. Ahn S, Lee SK: Diffusion tensor imaging: exploring the motor networks and clinical applications. Korean J Radiol 12:651661, 2011

2. Behrens TE, Johansen-Berg H, Woolrich MW, Smith SM, Wheeler-Kingshott CA, Boulby PA, et al: Non-invasive mapping of connections between human thalamus and cortex using diffusion imaging. Nat Neurosci 6:750-757, 2003

3. Bello L, Castellano A, Fava E, Casaceli G, Riva M, Scotti G, et al: Intraoperative use of diffusion tensor imaging fiber tractography and subcortical mapping for resection of gliomas: technical considerations. Neurosurg Focus 28(2):E6, 2010

4. Berman JI, Berger MS, Mukherjee P, Henry RG: Diffusiontensor imaging-guided tracking of fibers of the pyramidal tract combined with intraoperative cortical stimulation mapping in patients with gliomas. J Neurosurg 101:66-72, 2004

5. Byrnes TJ, Barrick TR, Bell BA, Clark CA: Diffusion tensor imaging discriminates between glioblastoma and cerebral metastases in vivo. NMR Biomed 24:54-60, 2011

6. Catani M: From hodology to function. Brain 130:602-605, 2007

7. Catani M, Howard RJ, Pajevic S, Jones DK: Virtual in vivo interactive dissection of white matter fasciculi in the human brain. Neuroimage 17:77-94, 2002

8. Chen F, Zhang X, Li M, Wang R, Wang HT, Zhu F, et al: Axial diffusivity and tensor shape as early markers to assess cerebral white matter damage caused by brain tumors using quantitative diffusion tensor tractography. CNS Neurosci Ther 18: 667-673, 2012

9. Chenevert TL, Brunberg JA, Pipe JG: Anisotropic diffusion in human white matter: demonstration with MR techniques in vivo. Radiology 177:401-405, 1990

10. Coenen VA, Krings T, Mayfrank L, Polin RS, Reinges MH, Thron A, et al: Three-dimensional visualization of the pyramidal tract in a neuronavigation system during brain tumor surgery: first experiences and technical note. Neurosurgery 49:86-93, 2001

11. Conturo TE, Lori NF, Cull TS, Akbudak E, Snyder AZ, Shimony JS, et al: Tracking neuronal fiber pathways in the living human brain. Proc Natl Acad Sci U S A 96:10422-10427, 1999

12. Dorward NL, Alberti O, Velani B, Gerritsen FA, Harkness WF, Kitchen ND, et al: Postimaging brain distortion: magnitude, correlates, and impact on neuronavigation. J Neurosurg 88:656-662, 1998

13. Duffau H: The anatomo-functional connectivity of language revisited. New insights provided by electrostimulation and tractography. Neuropsychologia 46:927-934, 2008

14. Duffau H: Introduction. Surgery of gliomas in eloquent areas: from brain hodotopy and plasticity to functional neurooncology. Neurosurg Focus 28(2):Intro, 2010

15. Duffau H: A plea to pay more attention on the anatomo-functional connectivity in surgical management of brain cavernomas. World Neurosurg [epub ahead of print], 2012

16. Duffau H, Capelle L, Denvil D, Sichez N, Gatignol P, Taillandier L, et al: Usefulness of intraoperative electrical subcortical mapping during surgery for low-grade gliomas located within eloquent brain regions: functional results in a consecutive series of 103 patients. J Neurosurg 98:764-778, 2003

17. ffytche DH, Catani M: Beyond localization: from hodology to function. Philos Trans R Soc Lond B Biol Sci 360:767-779, 2005

18. Giussani C, Poliakov A, Ferri RT, Plawner LL, Browd SR, Shaw DW, et al: DTI fiber tracking to differentiate demyelinating diseases from diffuse brain stem glioma. Neuroimage 52:217-223, 2010

19. González-Darder JM, González-López P, Talamantes F, Quilis V, Cortés V, García-March G, et al: Multimodal navigation in the functional microsurgical resection of intrinsic brain tumors located in eloquent motor areas: role of tractography. Neurosurg Focus 28(2):E5, 2010

20. Gössl C, Fahrmeir L, Pütz B, Auer LM, Auer DP: Fiber tracking from DTI using linear state space models: detectability of the pyramidal tract. Neuroimage 16:378-388, 2002

21. Gupta A, Shah A, Young RJ, Holodny AI: Imaging of brain tumors: functional magnetic resonance imaging and diffusion tensor imaging. Neuroimaging Clin N Am 20:379-400, 2010

22. Henry RG, Berman JI, Nagarajan SS, Mukherjee P, Berger MS: Subcortical pathways serving cortical language sites: initial experience with diffusion tensor imaging fiber tracking combined with intraoperative language mapping. Neuroimage $21: 616-622,2004$

23. Hua C, Merchant TE, Gajjar A, Broniscer A, Zhang Y, Li Y, et al: Brain tumor therapy-induced changes in normal-appearing brainstem measured with longitudinal diffusion tensor imaging. Int J Radiat Oncol Biol Phys 82:2047-2054, 2012

24. Ius T, Isola M, Budai R, Pauletto G, Tomasino B, Fadiga L, et al: Low-grade glioma surgery in eloquent areas: volumetric analysis of extent of resection and its impact on overall survival. A single-institution experience in 190 patients. Clinical article. J Neurosurg 117:1039-1052, 2012

25. Jellison BJ, Field AS, Medow J, Lazar M, Salamat MS, Alexander AL: Diffusion tensor imaging of cerebral white matter: a pictorial review of physics, fiber tract anatomy, and tumor imaging patterns. AJNR Am J Neuroradiol 25:356-369, 2004

26. Jolapara M, Patro SN, Kesavadas C, Saini J, Thomas B, Gupta $\mathrm{AK}$, et al: Can diffusion tensor metrics help in preoperative grading of diffusely infiltrating astrocy tomas? A retrospective study of 36 cases. Neuroradiology 53:63-68, 2011 
27. Kamada K, Todo T, Masutani Y, Aoki S, Ino K, Takano T, et al: Combined use of tractography-integrated functional neuronavigation and direct fiber stimulation. J Neurosurg 102: 664-672, 2005

28. Liu X, Tian W, Kolar B, Yeaney GA, Qiu X, Johnson MD, et al: MR diffusion tensor and perfusion-weighted imaging in preoperative grading of supratentorial nonenhancing gliomas. Neuro Oncol 13:447-455, 2011

29. Lober RM, Guzman R, Cheshier SH, Fredrick DR, Edwards MS, Yeom KW: Application of diffusion tensor tractography in pediatric optic pathway glioma. Clinical article. J Neurosurg Pediatr 10:273-280, 2012

30. Moseley ME, Cohen Y, Kucharczyk J, Mintorovitch J, Asgari HS, Wendland MF, et al: Diffusion-weighted MR imaging of anisotropic water diffusion in cat central nervous system. Radiology 176:439-445, 1990

31. Mukherjee P, Berman JI, Chung SW, Hess CP, Henry RG: Diffusion tensor MR imaging and fiber tractography: theoretic underpinnings. AJNR Am J Neuroradiol 29:632-641, 2008

32. Nickerson JP, Salmela MB, Koski CJ, Andrews T, Filippi CG: Diffusion tensor imaging of the pediatric optic nerve: intrinsic and extrinsic pathology compared to normal controls. J Magn Reson Imaging 32:76-81, 2010

33. Nimsky C, Ganslandt O, Fahlbusch R: Implementation of fiber tract navigation. Neurosurgery 58 (4 Suppl 2):ONS292ONS304, 2006

34. Nimsky C, Ganslandt O, Hastreiter P, Wang R, Benner T, Sorensen AG, et al: Preoperative and intraoperative diffusion tensor imaging-based fiber tracking in glioma surgery. Neurosurgery 56:130-138, 2005

35. Nimsky C, Ganslandt O, Merhof D, Sorensen AG, Fahlbusch $\mathrm{R}$ : Intraoperative visualization of the pyramidal tract by diffusion-tensor-imaging-based fiber tracking. Neuroimage 30: 1219-1229, 2006

36. Nossek E, Korn A, Shahar T, Kanner AA, Yaffe H, Marcovici $\mathrm{D}$, et al: Intraoperative mapping and monitoring of the corticospinal tracts with neurophysiological assessment and 3-dimensional ultrasonography-based navigation. Clinical article. J Neurosurg 114:738-746, 2011

37. Ohue S, Kohno S, Inoue A, Yamashita D, Harada H, Kumon $Y$, et al: Accuracy of diffusion tensor magnetic resonance imaging-based tractography for surgery of gliomas near the pyramidal tract: a significant correlation between subcortical electrical stimulation and postoperative tractography. Neurosurgery 70:283-294, 2012

38. Papadakis NG, Xing D, Huang CL, Hall LD, Carpenter TA: A comparative study of acquisition schemes for diffusion tensor imaging using MRI. J Magn Reson 137:67-82, 1999

39. Provenzale JM, McGraw P, Mhatre P, Guo AC, Delong D: Peritumoral brain regions in gliomas and meningiomas: investigation with isotropic diffusion-weighted MR imaging and diffusion-tensor MR imaging. Radiology 232:451-460, 2004

40. Robles SG, Gatignol P, Lehéricy S, Duffau H: Long-term brain plasticity allowing a multistage surgical approach to World Health Organization Grade II gliomas in eloquent areas. Report of 2 cases. J Neurosurg 109:615-624, 2008

41. Saksena S, Jain R, Narang J, Scarpace L, Schultz LR, Lehman NL, et al: Predicting survival in glioblastomas using diffusion tensor imaging metrics. J Magn Reson Imaging 32:788-795, 2010

42. Schaefer PW, Grant PE, Gonzalez RG: Diffusion-weighted MR imaging of the brain. Radiology 217:331-345, 2000

43. Setzer M, Murtagh RD, Murtagh FR, Eleraky M, Jain S, Marquardt G, et al: Diffusion tensor imaging tractography in patients with intramedullary tumors: comparison with intraoperative findings and value for prediction of tumor resectability. Clinical article. J Neurosurg Spine 13:371-380, 2010
44. Stieltjes B, Schlüter M, Didinger B, Weber MA, Hahn HK, Parzer $\mathrm{P}$, et al: Diffusion tensor imaging in primary brain tumors: reproducible quantitative analysis of corpus callosum infiltration and contralateral involvement using a probabilistic mixture model. Neuroimage 31:531-542, 2006

45. Sun GC, Chen XL, Zhao Y, Wang F, Hou BK, Wang YB, et al: Intraoperative high-field magnetic resonance imaging combined with fiber tract neuronavigation-guided resection of cerebral lesions involving optic radiation. Neurosurgery 69:1070-1084, 2011

46. Toh CH, Castillo M, Wong AM, Wei KC, Wong HF, Ng SH, et al: Primary cerebral lymphoma and glioblastoma multiforme: differences in diffusion characteristics evaluated with diffusion tensor imaging. AJNR Am J Neuroradiol 29:471-475, 2008

47. Van Essen DC, Ugurbil K: The future of the human connectome. Neuroimage 62:1299-1310, 2012

48. Wang S, Kim S, Chawla S, Wolf RL, Knipp DE, Vossough A, et al: Differentiation between glioblastomas, solitary brain metastases, and primary cerebral lymphomas using diffusion tensor and dynamic susceptibility contrast-enhanced MR imaging. AJNR Am J Neuroradiol 32:507-514, 2011

49. Wang S, Kim S, Chawla S, Wolf RL, Zhang WG, O'Rourke DM, et al: Differentiation between glioblastomas and solitary brain metastases using diffusion tensor imaging. Neuroimage 44:653-660, 2009

50. Wu JS, Zhou LF, Tang WJ, Mao Y, Hu J, Song YY, et al: Clinical evaluation and follow-up outcome of diffusion tensor imaging-based functional neuronavigation: a prospective, controlled study in patients with gliomas involving pyramidal tracts. Neurosurgery 61:935-949, 2007

51. Xu JL, Li YL, Lian JM, Dou SW, Yan FS, Wu H, et al: Distinction between postoperative recurrent glioma and radiation injury using MR diffusion tensor imaging. Neuroradiology 52:1193-1199, 2010

52. Yamada K, Kizu O, Mori S, Ito H, Nakamura H, Yuen S, et al: Brain fiber tracking with clinically feasible diffusion-tensor MR imaging: initial experience. Radiology 227:295-301, 2003

53. Yogarajah M, Focke NK, Bonelli SB, Thompson P, Vollmar C, McEvoy AW, et al: The structural plasticity of white matter networks following anterior temporal lobe resection. Brain 133:2348-2364, 2010

54. Yordanova YN, Moritz-Gasser S, Duffau H: Awake surgery for WHO Grade II gliomas within "noneloquent" areas in the left dominant hemisphere: toward a "supratotal" resection. Clinical article. J Neurosurg 115:232-239, 2011

55. Zhu FP, Wu JS, Song YY, Yao CJ, Zhuang DX, Xu G, et al: Clinical application of motor pathway mapping using diffusion tensor imaging tractography and intraoperative direct subcortical stimulation in cerebral glioma surgery: a prospective cohort study. Neurosurgery 71:1170-1184, 2012

56. Zou QG, Xu HB, Liu F, Guo W, Kong XC, Wu Y: In the assessment of supratentorial glioma grade: the combined role of multivoxel proton MR spectroscopy and diffusion tensor imaging. Clin Radiol 66:953-960, 2011

Manuscript submitted December 15, 2012.

Accepted January 23, 2013.

Please include this information when citing this paper: DOI: 10.3171/2013.1.FOCUS12412.

Address correspondence to: Steven Brem, M.D., Department of Neurosurgery, Hospital of the University of Pennsylvania, 3400 Spruce Street, 3rd Floor Silverstein, Philadelphia, Pennsylvania 19104.email: steven.brem@uphs.upenn.edu. 